\title{
Frequency characteristic of a uniformly rotating non-dithered laser gyro in exact and polynomial forms
}

This paper was downloaded from TechRxiv (https://www.techrxiv.org).

LICENSE

CC BY 4.0

SUBMISSION DATE / POSTED DATE

$29-12-2021 / 11-01-2022$

CITATION

Bondarenko, Evgeny (2022): Frequency characteristic of a uniformly rotating non-dithered laser gyro in exact and polynomial forms. TechRxiv. Preprint. https://doi.org/10.36227/techrxiv.17702315.v1

DOI

10.36227/techrxiv.17702315.v1 


\title{
Frequency characteristic of a uniformly rotating
}

\section{non-dithered laser gyro in exact and polynomial forms}

\author{
Evgeny A. Bondarenko
}

Abstract-In some inertial navigation systems of a carousel type, laser gyros operate in regime of uniform rotation (their monoblocks do not dither). For development of such systems and computer simulation of their work, one needs to have the "exact" (in wide range of angular velocities $\Omega$ ) analytical expression for counterpropagating waves beat frequency $\omega_{\text {beat }}=\omega_{\text {beat }}(\Omega)$ of uniformly rotating device. This expression may be obtained by solving the well-known system of laser gyro dynamic equations with accuracy to second order in parameters of counterpropagating waves linear coupling. But before use of such non-dithered laser gyros in the named inertial system, their metrological parameters (scale factors and null shifts) must be preliminary calibrated. For synthesis and qualitative analysis of methodical errors of the procedure of such calibration on a quickly rotating platform of a single-axis stand, one needs also to have the approximate analytical expression for counterpropagating waves beat frequency in the form of polynomial $\omega_{\text {beat }}^{P}=K_{(1)} \Omega+K_{(0)}+K_{(-1)} \Omega^{-1}+K_{(-2)} \Omega^{-2}+K_{(-3)} \Omega^{-3}+K_{(-4)} \Omega^{-4}$ with coefficients $K_{(1)}, \ldots, K_{(-4)}$ which involve parameters of the exact expression for $\omega_{\text {beat }}$. As analysis of the literature shows, the known expression for $\omega_{\text {beat }}$ and the known relations for some of coefficients of polynomial $\omega_{\text {beat }}^{P}$ are not complete and, therefore, must be modified. In the paper, the result of such modification is presented.

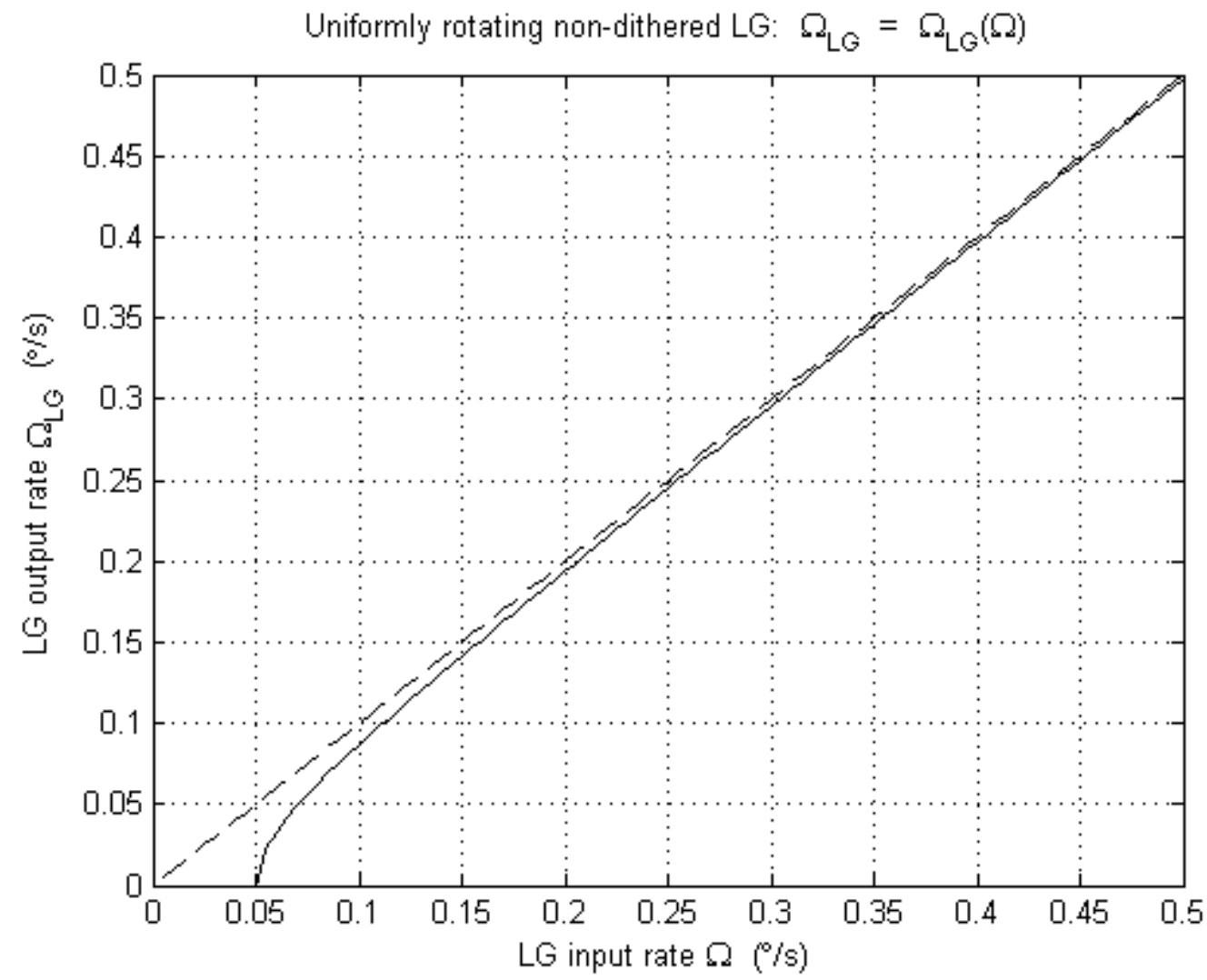

Fragment of output characteristic of a uniformly rotating non-dithered laser gyro (LG)

Index Terms_ring laser gyroscope, ring gas laser, frequency characteristic. 


\section{INTRODUCTION}

A mong the main types of laser gyros that are widely used in practice, one can highlight a device on a base of a ring $\mathrm{He}-\mathrm{Ne}$ gas laser $\left({ }^{20} \mathrm{Ne}:{ }^{22} \mathrm{Ne}=1: 1\right)$ with a planar $\mathrm{N}$-mirror $(N=3,4)$ resonator which provides radiation linearly polarized in the sagittal plane. Pumping of the laser which operates, as a rule, at wavelength $\lambda_{0}=0.6328 \times 10^{-6} \mathrm{~m}$, is realized by means of a constant discharge current with use a symmetric scheme: one cathode - two anodes [1]-[5].

According to relations (6.45)-(6.47) from [6], the system of equations describing the dynamics of dimensionless intensities $I_{j} \quad(j=1,2)$ and phase difference $\psi$ of counterpropagating waves of such laser gyro (under condition of equal currents in its discharge legs) may be presented in the form

$$
\begin{aligned}
\dot{I}_{1} & =\left(\alpha_{1}-\beta I_{1}-\theta I_{2}\right) I_{1}-2 r_{2}\left(I_{1} I_{2}\right)^{1 / 2} \cos \left(\psi+\varepsilon_{2}\right), \\
\dot{I}_{2} & =\left(\alpha_{2}-\beta I_{2}-\theta I_{1}\right) I_{2}-2 r_{1}\left(I_{1} I_{2}\right)^{1 / 2} \cos \left(\psi-\varepsilon_{1}\right), \\
\dot{\psi} & =M \Omega+(\rho-\tau)\left(I_{1}-I_{2}\right) \\
& +r_{2}\left(I_{2} / I_{1}\right)^{1 / 2} \sin \left(\psi+\varepsilon_{2}\right)+r_{1}\left(I_{1} / I_{2}\right)^{1 / 2} \sin \left(\psi-\varepsilon_{1}\right) .
\end{aligned}
$$

In deriving these equations it was taken into account that the electromagnetic wave with $j=1$ propagates in the direction of the laser gyro rotation.

In system (1): $\alpha_{j}, \beta, \theta, \rho, \tau$ are the Lamb coefficients which characterize the properties of the active medium; $M=\left(1+K_{a}\right) M_{g}$ is the laser gyro scale multiplier which is determined mainly by its geometrical component $M_{g}=8 \pi A /\left(\lambda_{0} L\right) \quad(L-$ axis contour perimeter, $A$ - area enclosed by axis contour) but which takes into account also the properties of the active medium ([7]) by means of very small parameter $K_{a}\left(K_{a}<0,\left|K_{a}\right| \ll<1\right) ; \Omega$ is the angular velocity with which the laser gyro rotates in the inertial space; $r_{j}$ and $\varepsilon_{j}$ are the modules and arguments of complex integral coefficients $r_{j} \exp \left\{\varepsilon_{j}\right\}$ of counterpropagating waves linear coupling, characterizing their interaction through backscattering, absorption, and transmission of radiation on the mirrors.

In some inertial navigation systems of a carousel type (see paragraphs 3.7.5 and 3.8.7 in [5]), laser gyros operate in regime of uniform rotation (their monoblocks do not dither). For development of such systems and computer simulation of their work, one needs to have the "exact" (in wide range of $\Omega$ ) analytical expression for counterpropagating waves beat frequency $\omega_{\text {beat }}$ of uniformly rotating device. Such expression may be obtained as a result of solving system (1) with accuracy to second order in parameters $r_{j}$ of

Manuscript received September 12, 2021.

The author is with the National Technical University of Ukraine "Igor Sikorsky Kyiv Polytechnic Institute", Kyiv, Ukraine (e-mail: ea_bndrk@ukr.net). counterpropagating waves linear coupling. If relation $\omega_{\text {beat }}=\omega_{\text {beat }}(\Omega)$ is known, then the number of information pulses $\Delta N$, accumulated on the laser gyro output during time $\Delta t$, can be found from the differential equation

$$
\frac{d N}{d t}=\frac{k_{f}}{2 \pi} \omega_{\text {beat }},
$$

where $d N / d t$ is the pulse repetition rate on the gyro output, and $k_{f}$ is the "frequency multiplication coefficient" (as a rule, $\left.k_{f}=1,2,4\right)$. In the literature, equation (2) is called "frequency characteristic of a uniformly rotating laser gyro".

But before use of such non-dithered laser gyros in the named inertial system, their metrological parameters (scale factors and null shifts) must be preliminary calibrated (see item $b$ of point 12.9.3.1 in [8]). For synthesis and qualitative analysis of methodical errors of the procedure of such calibration on a quickly rotating platform of a single-axis stand, one needs also to have the approximate analytical expression for counterpropagating waves beat frequency in asymptotic limit of high values of $\Omega$. This relation may be taken in the form of polynomial

$\omega_{\text {beat }}^{P}=K_{(1)} \Omega+K_{(0)}+K_{(-1)} \Omega^{-1}+K_{(-2)} \Omega^{-2}+K_{(-3)} \Omega^{-3}+K_{(-4)} \Omega^{-4}$

with coefficients $K_{(1)}, \ldots, K_{(-4)}$ which involve parameters of the exact expression for $\omega_{\text {beat }}$.

\section{A. Results of analysis of the literature}

\section{Known exact expression for $\omega_{\text {beat }}$}

As analysis of the literature shows, the known expression for $\omega_{\text {beat }}$ (calculated to second order in parameters $r_{j}$ of counterpropagating waves linear coupling) has the form

$$
\begin{aligned}
\omega_{\text {beat }} & =\omega_{0}+\left[1-\frac{\omega_{s(0)}^{2}}{2 \omega^{2}}+\frac{\left(1+4 T^{2}\right) r_{m}^{2}}{2\left(\alpha_{m}^{2}+\omega^{2}\right)}\right] \omega \\
& +D\left(r_{2}^{2}-r_{1}^{2}\right)\left\{-\frac{1}{\omega^{2}}+\frac{1}{\alpha_{m}^{2}+\omega^{2}}\left[1+\frac{\alpha_{m}\left(\alpha_{p}+\alpha_{m}\right)}{2\left(\alpha_{p}^{2}+\omega^{2}\right)}\right]\right. \\
& \left.-\frac{2 T^{2} \alpha_{m}^{2}}{\omega^{2}\left(\alpha_{m}^{2}+\omega^{2}\right)}\left[1+\frac{\alpha_{p}}{\alpha_{m}} \frac{\alpha_{p} \alpha_{m}-\omega^{2}}{\alpha_{p}^{2}+\omega^{2}}\right]\right\} \omega .
\end{aligned}
$$

The first term in the right-hand side of (4) is well-known (see, for example, [1], [6], [9], [10]). The second term is known from [10] (see formulas (6.31), (6.32), (6.7) therein). And the third term is known from [11] (see expression (23) therein).

As one can see, the second and the third terms in (4) describe only the reversible (with respect to $\Omega$ ) components of $\omega_{\text {beat }}$. There are not the nonreversible ones in (4).

NOTE: $\triangleleft$ In the literature, in works [11], [12], [13], in addition to (4), there are correspondingly three qualitatively different versions of expressions for the nonreversible components of $\omega_{\text {beat }}$. It is important to note that these relations are obtained with accuracy to the fourth order in parameters $r_{j}$. Their common feature is that they are proportional to the combination $\left(r_{2}^{2}-r_{1}^{2}\right) r_{1} r_{2} \sin \varepsilon_{12}$ which describes the influence of the factor of asymmetry $\left(r_{1} \neq r_{2}\right)$ of counterpropagating 
waves linear coupling. As it will be shown below, such factor is less significant than another one - the factor of inequality $\left(\alpha_{1} \neq \alpha_{2}\right)$ of counterpropagating waves amplification due to nonreciprocal resonator losses. This more important factor will manifest itself already to second order in parameters $r_{j}$.

$\triangleright$

In (4), the following notations are used:

$\omega=M \Omega$ is the laser gyro counterpropagating waves frequencies splitting caused by its rotation in the inertial space with angular velocity $\Omega$ (in ideal device $\omega_{\text {beat }}=\omega=M \Omega$ );

$\varepsilon_{12}=\varepsilon_{1}+\varepsilon_{2}$ is the sum of arguments of complex integral coefficients $r_{j} \exp \left\{\varepsilon_{j}\right\}$ of counterpropagating waves linear coupling;

$r_{p}=\left(r_{1}^{2}+r_{2}^{2}+2 r_{1} r_{2} \cos \varepsilon_{12}\right)^{1 / 2}$ and $r_{m}=\left(r_{1}^{2}+r_{2}^{2}-2 r_{1} r_{2} \cos \varepsilon_{12}\right)^{1 / 2}$ are the combinations of modules and arguments of these coefficients;

$\alpha_{p}=\alpha=(1 / 2)\left(\alpha_{1}+\alpha_{2}\right)$ is the inverse relaxation time of the sum of counterpropagating waves intensities;

$\alpha_{m}=\alpha_{p}(1-h) /(1+h)$ is the inverse relaxation time of the difference of counterpropagating waves intensities. Here $h=\theta / \beta$ is the dimensionless parameter which depends linearly on the $\mathrm{He}-\mathrm{Ne}$ mixture total pressure;

$D=(1 / 2) \alpha_{m}^{-1}\left(\alpha_{2}-\alpha_{1}\right) \quad$ is the small dimensionless parameter which characterizes the degree of inequality of counterpropagating waves amplification caused by difference of the laser gyro resonator losses. In ideal device, the losses for both waves are equal, and $D=0$;

$T=(\rho-\tau) /(\beta-\theta)$ is the small dimensionless parameter which characterizes the degree of laser gyro resonator frequency detuning from the center of the active medium emission line. This detuning is caused by a small (with respect to $\lambda_{0}$ ) systematic error $\Delta L$ of the perimeter control extremum system (its periodical search steps are not considered). In ideal case of accurate laser gyro resonator frequency tuning to the line center, $\Delta L=0, \rho=\tau=0$, and $T=0$

$\omega_{0}=-2 T D \alpha_{m}=-T\left(\alpha_{2}-\alpha_{1}\right)$ is the so-called "null shift" of the laser gyro frequency characteristic. In other words, it is the counterpropagating waves frequencies splitting (even when $\Omega=0$ ) caused by a multiplicative interaction of the factor of unequal waves amplification and the factor of resonator frequency detuning. If quantity $\omega_{0}$ is known, then the laser gyro null shift $\Omega_{0}$, which has dimension of angular velocity, may be calculated as $\Omega_{0}=\omega_{0} / M$;

$\omega_{s(0)}=\left(r_{p}^{2}+8 T r_{1} r_{2} \sin \varepsilon_{12}+4 T^{2} r_{m}^{2}\right)^{1 / 2}$ is the parameter which has dimension of angular frequency and characterizes the halfwidth of synchronization zone of the laser gyro counterpropagating waves frequencies in approximation $D=0$. If quantity $\omega_{s(0)}$ is known, then the laser gyro synchronization zone halfwidth $\Omega_{s(0)}$, which has dimension of angular velocity, may be calculated in such approximation as $\Omega_{s(0)}=\omega_{s(0)} / M$.

NOTE: $\triangleleft$ In the case $D=T=0$, expression (4) has the form $\omega_{\text {beat }}=\left\{1-r_{p}^{2} /\left(2 \omega^{2}\right)+r_{m}^{2} /\left[2\left(\alpha_{m}^{2}+\omega^{2}\right)\right]\right\} \omega$ which is confirmed theoretically and experimentally in [14]. $\triangleright$

\section{Known expressions for coefficients of polynomial $\omega_{\text {beat }}^{P}$}

As analysis of the literature shows, the known relations for coefficients $K_{(1)}, \ldots, K_{(-4)}$ of polynomial $\omega_{\text {beat }}^{P}$ (calculated to second order in parameters $r_{j}$ ) have the following forms:

Coefficients $K_{(1)}$ and $K_{(0)}$. As it follows directly from (4),

$$
K_{(1)}=M,
$$

and, in accordance, for example, with [1], [6], [9], [10],

$$
K_{(0)}=\omega_{0}, \quad \omega_{0}=-T\left(\alpha_{2}-\alpha_{1}\right), \quad T=(\rho-\tau) /(\beta-\theta) .
$$

Coefficients $K_{(-1)}$ and $K_{(-3)}$. According to [9], [10], [15]-[17],

$$
K_{(-1)}=-2 r_{1} r_{2}\left(\cos \varepsilon_{12}+2 T \sin \varepsilon_{12}\right) / M,
$$

and, in accordance with [17],

$$
K_{(-3)}=-(1 / 2)\left(1+4 T^{2}\right) \alpha_{m}^{2} r_{m}^{2} / M^{3} .
$$

Coefficients $K_{(-2)}$ and $K_{(-4)}$. In the literature, there are not expressions for these coefficients calculated to second order in parameters $r_{j}$. So the corresponding relations for these quantities must be found.

NOTE: $\triangleleft$ In the literature ([11], [12], [13]), there are qualitatively different expressions for these coefficients but calculated to the fourth order in parameters $r_{j}$.

\section{B. Principal goals of this paper}

As analysis of the literature shows, the known exact expression (4) for counterpropagating waves beat frequency $\omega_{\text {beat }}$ and the known relation (8) for coefficient $K_{(-3)}$ of polynomial $\omega_{\text {beat }}^{P}$ are not complete: they do not reflect fully the influence of the factor of inequality $\left(\alpha_{1} \neq \alpha_{2}\right)$ of counterpropagating waves amplification due to nonreciprocal resonator losses. Moreover, the sought for (to second order in parameters $r_{j}$ ) expressions for coefficients $K_{(-2)}$ and $K_{(-4)}$ of $\omega_{\text {beat }}^{P}$ are still unknown. So the principal goals of this paper are: 1) to propose the modified exact expression for $\omega_{\text {beat }} ; 2$ ) to confirm the known relations for coefficients $K_{(1)}, K_{(0)}$, and $K_{(-1)}$ of polynomial $\left.\omega_{\text {beat }}^{P} ; 3\right)$ to present the modified expression for coefficient $\left.K_{(-3)} ; 4\right)$ to find the sought for relations for coefficients $K_{(-2)}$ and $K_{(-4)}$. 


\section{MODIFIED EXACT EXPRESSION FOR $\omega_{\text {beat }}$}

According to the author's report [18], the modified exact expression for $\omega_{\text {beat }}$ may be written in the form

$$
\begin{aligned}
& \omega_{\text {beat }}=\omega_{0}+\left[1-\frac{\omega_{s(0)}^{2}}{2 \omega^{2}}+\frac{\left(1+4 T^{2}\right) r_{m}^{2}}{2\left(\alpha_{m}^{2}+\omega^{2}\right)}\right] \omega \\
& +D\left(r_{2}^{2}-r_{1}^{2}\right)\left\{-\frac{1}{\omega^{2}}+\frac{1}{\alpha_{m}^{2}+\omega^{2}}\left[1+\frac{\alpha_{m}\left(\alpha_{p}+\alpha_{m}\right)}{2\left(\alpha_{p}^{2}+\omega^{2}\right)}\right]\right. \\
& \left.-\frac{2 T^{2} \alpha_{m}^{2}}{\omega^{2}\left(\alpha_{m}^{2}+\omega^{2}\right)}\left[1+\frac{\alpha_{p}}{\alpha_{m}} \frac{\alpha_{p} \alpha_{m}-\omega^{2}}{\alpha_{p}^{2}+\omega^{2}}\right]\right\} \omega \\
& +\left(1+4 T^{2}\right)\left(\alpha_{2}-\alpha_{1}\right) r_{1} r_{2} \sin \varepsilon_{12} \frac{\alpha_{p} \alpha_{m}-\omega^{2}}{2\left(\alpha_{p}^{2}+\omega^{2}\right)\left(\alpha_{m}^{2}+\omega^{2}\right)} \\
& +\omega_{0}\left[\frac{\omega_{s(0)}^{2}}{2 \omega^{2}}+\left(1+4 T^{2}\right) r_{m}^{2} \frac{\alpha_{m}^{2}-\omega^{2}}{2\left(\alpha_{m}^{2}+\omega^{2}\right)^{2}}\right] .
\end{aligned}
$$

Expression (9) is derived on the base of system (1) with the help of the procedure which is generalization (for the case $T \neq 0$ ) of the method developed earlier by the author in [19] for the case $T=0$. The first, the second, and the third terms in (9) confirm the known expression (4) for $\omega_{\text {beat }}$. But the fourth and the fifth terms in (9) are substantially new. They describe two nonreversible with respect to $\Omega$ components of $\omega_{\text {beat }}$ caused by the factor of inequality of counterpropagating waves amplification due to nonreciprocal resonator losses.

Expression (9) may be used in the range $\left[\Omega \leq-\left(3 \Omega_{s}+\Omega_{0}\right)\right.$; $\left.\Omega \geq+\left(3 \Omega_{s}-\Omega_{0}\right)\right]$, where $\Omega_{0}=\omega_{0} / M$, and

$\Omega_{s}=\left[r_{p}^{2}+8 T r_{1} r_{2} \sin \varepsilon_{12}+4 T^{2} r_{m}^{2}+2 D\left(r_{2}^{2}-r_{1}^{2}\right)\left(1+4 T^{2}\right)\right]^{1 / 2} / M$

is the laser gyro synchronization zone halfwidth calculated for general case $D \neq 0$.

Expression (9) is valid, if the condition of weakness of counterpropagating waves linear coupling is fulfilled. It implies that for all given possible values of laser gyro total discharge current, the values of ratios $r_{p} / \alpha_{p}$ and $r_{m} / \alpha_{m}$ must be much less than unity. In modern devices, operating with sufficiently high level of pumping (see paragraph 3.3.2 in [5]), the named condition, as a rule, is satisfied.

NOTE: $\triangleleft$ For the laser gyro (with a four-mirror square resonator) operating at total pressures of the $\mathrm{He}-\mathrm{Ne}$ mixture from 1 to 5-6 Torr, a set of engineer formulas for calculation of the parameters $\alpha_{j}, \beta, \theta, \rho, \tau, K_{a}$, and $r_{j}, \varepsilon_{j}$ (case $r_{1}=r_{2}=r, \varepsilon_{1}=\varepsilon_{2}=\varepsilon$ ) of system (1) is proposed in [20]. A set of relations for estimating the parameters $r_{j}, \varepsilon_{j}$ of such laser gyro for general case $\left(r_{1} \neq r_{2}, \varepsilon_{1} \neq \varepsilon_{2}\right)$ is presented in [21]. Formulas for simulating the dynamics of the parameters $r_{j}, \varepsilon_{j}$ during the device operation in the self-heating regime are proposed in [22]. $\triangleright$

\section{MODIFIED EXPRESSIONS FOR COEFFICIENTS OF POLYNOMIAL $\omega_{\text {beat }}^{P}$}

The modified expressions for coefficients $K_{(1)}, \ldots, K_{(-4)}$ of polynomial $\omega_{\text {beat }}^{P}$ may be obtained on the base of relation (9) for $\omega_{\text {beat }}$ with the help of the following approximate formulas (which are valid for $|\omega| \gg \alpha_{p}, \alpha_{m}$ ):

$$
\begin{aligned}
& F_{1}=\omega /\left(\alpha_{m}^{2}+\omega^{2}\right) \approx 1 / \omega-\alpha_{m}^{2} / \omega^{3}, \\
& F_{2}=1 /\left[\omega\left(\alpha_{m}^{2}+\omega^{2}\right)\right] \approx 1 / \omega^{3}, \\
& F_{3}=1 /\left(\alpha_{m}^{2}+\omega^{2}\right) \approx 1 / \omega^{2}-\alpha_{m}^{2} / \omega^{4}, \\
& F_{4}=\omega^{2} /\left(\alpha_{m}^{2}+\omega^{2}\right) \approx 1-\alpha_{m}^{2} / \omega^{2}+\alpha_{m}^{4} / \omega^{4}, \\
& F_{5}=1 /\left(\alpha_{m}^{2}+\omega^{2}\right)^{2} \approx 1 / \omega^{4}, \\
& F_{6}=\omega^{2} /\left(\alpha_{m}^{2}+\omega^{2}\right)^{2} \approx 1 / \omega^{2}-2 \alpha_{m}^{2} / \omega^{4}, \\
& F_{7}=\omega /\left[\left(\alpha_{p}^{2}+\omega^{2}\right)\left(\alpha_{m}^{2}+\omega^{2}\right)\right] \approx 1 / \omega^{3}, \\
& F_{8}=1 /\left[\omega\left(\alpha_{p}^{2}+\omega^{2}\right)\left(\alpha_{m}^{2}+\omega^{2}\right)\right] \approx 0, \\
& F_{9}=1 /\left[\left(\alpha_{p}^{2}+\omega^{2}\right)\left(\alpha_{m}^{2}+\omega^{2}\right)\right] \approx 1 / \omega^{4}, \\
& F_{10}=\omega^{2} /\left[\left(\alpha_{p}^{2}+\omega^{2}\right)\left(\alpha_{m}^{2}+\omega^{2}\right)\right] \approx 1 / \omega^{2}-\left(\alpha_{p}^{2}+\alpha_{m}^{2}\right) / \omega^{4} .
\end{aligned}
$$

Taking into account $\omega=M \Omega$, after substituting (11) into (9) and collecting the corresponding terms, we obtain ([23]):

$$
\begin{aligned}
K_{(1)}= & M, \\
K_{(0)}= & \omega_{0}, \quad \omega_{0}=-T\left(\alpha_{2}-\alpha_{1}\right), \quad T=(\rho-\tau) /(\beta-\theta), \\
K_{(-1)}= & -2 r_{1} r_{2}\left(\cos \varepsilon_{12}+2 T \sin \varepsilon_{12}\right) / M, \\
K_{(-3)}= & -(1 / 2)\left(1+4 T^{2}\right)\left[\alpha_{m}^{2} r_{m}^{2}\right. \\
& \left.-(1 / 2)\left(\alpha_{p}-\alpha_{m}\right)\left(\alpha_{2}-\alpha_{1}\right)\left(r_{2}^{2}-r_{1}^{2}\right)\right] / M^{3}, \\
K_{(-2)}= & \left\{\omega_{0}\left[2 r_{1} r_{2}\left(\cos \varepsilon_{12}+2 T \sin \varepsilon_{12}\right)\right]\right. \\
- & \left.(1 / 2)\left(1+4 T^{2}\right)\left(\alpha_{2}-\alpha_{1}\right) r_{1} r_{2} \sin \varepsilon_{12}\right\} / M^{2}, \\
K_{(-4)}= & (1 / 2)\left(1+4 T^{2}\right)\left[\omega_{0}\left(3 \alpha_{m}^{2} r_{m}^{2}\right)\right. \\
+ & \left.\left(\alpha_{p}^{2}+\alpha_{m}^{2}+\alpha_{p} \alpha_{m}\right)\left(\alpha_{2}-\alpha_{1}\right) r_{1} r_{2} \sin \varepsilon_{12}\right] / M^{4} .
\end{aligned}
$$

These relations are valid for $|\Omega| \gg \Omega_{\alpha_{p}}, \Omega_{\alpha_{m}}$, where $\Omega_{\alpha_{p}}=\alpha_{p} / M, \Omega_{\alpha_{m}}=\alpha_{m} / M$.

As one can see, expressions (12)-(14) and (5)-(7) for coefficients $K_{(1)}, K_{(0)}, K_{(-1)}$ of polynomial $\omega_{\text {beat }}^{P}$ do not differ: they are identical. Expression (15) for coefficient $K_{(-3)}$ only generalizes the known relation (8) for general case $\alpha_{1} \neq \alpha_{2}$. But expressions (16), (17) for coefficients $K_{(-2)}$, 
$K_{(-4)}$ are substantially new. According to (16), (17), and relation $\omega_{0}=-T\left(\alpha_{2}-\alpha_{1}\right)$ in (13), coefficients $K_{(-2)}$ and $K_{(-4)}$ depend on difference $\Delta \alpha=\alpha_{2}-\alpha_{1}$ caused by the factor of inequality of counterpropagating waves amplification due to nonreciprocal resonator losses.

\section{REFERENCES}

[1] F. Aronowitz, "The laser gyro," in Laser Applications, M. Ross, Ed. New York: Academic Press, 1971, vol. 1, pp. 133-200.

[2] W. W. Chow, J. Gea-Banacloche, L. M. Pedrotti, V. E. Sanders, W. Schleich, and M. O. Scully, "The ring laser gyro," Rev. Mod. Phys., vol.57, no. 1, pp. 61-104, 1985.

[3] J. R. Wilkinson, "Ring lasers," Prog. Quant. Electr., vol. 11, pp. 1-103, 1987.

[4] M. Faucheux, D. Fayoux, and J. J. Roland, "The ring laser gyro," J. Optics (Paris), vol. 19, no. 3, pp. 101-115, 1988.

[5] F. Aronowitz, "Fundamentals of the ring laser gyro," in Optical Gyros and their Application, RTO AGARDograph 339, D. Loukianov, R. Rodloff, H. Sorg, and B. Stieler, Eds. Neuilly-sur-Seine, France, 1999, pp. 3-1-3-45.

[6] L. N. Menegozzi and W. E. Lamb, Jr., "Theory of a Ring Laser," Phys. Rev. A, vol. 8, no. 4, pp. 2103-2125, 1973.

[7] F. Aronowitz, J. E. Killpatrick, and S. P. Callaghan, "Power-Dependent Correction to the Scale Factor in the Laser Gyro," IEEE J. Quantum Electron., vol. QE-10, no. 2, pp. 201-208, 1974.

[8] IEEE Standard Specification Format Guide and Test Procedure for Single-Axis Laser Gyros (IEEE Std 647-2006).

[9] P. S. Landa and E. G. Lariontsev, "Regimes of beating and synchronization of counterpropagating waves in a rotating ring gas laser," Radiotekhn. Elektron., vol. 15, no. 6, pp. 1214-1226, 1970 (in Russian).

[10] S. G. Zeiger, Yu. L. Klimontovich, P. S. Landa, E. G. Lariontsev, and E. E. Fradkin, Wave and fluctuation processes in lasers. Moscow: Nauka, 1974 (in Russian).

[11] A. Ya. Birman, P. B. Naumov, and A. F. Savushkin, "Nonreversible component of the frequency characteristic of a ring laser,". Sov. J. Quantum Electron., vol. 11, no. 11, pp. 1498-1501, 1981.

[12] V. A. Markelov, "Frequency response of a ring laser with a reversible pedestal," Sov. J. Quantum Electron., vol. 9, no. 8, pp. 1057-1059, 1979.

[13] Yu. A. Mamaev, "On nonreversible component of the frequency difference of a laser gyroscope," Opt. Spektrosk., vol. 63, no. 6, pp. 1388-1389, 1987 (in Russian).

[14] F. Aronowitz and W. L. Lim, "Positive scale factor correction in the laser gyro," IEEE J. Quantum Electron., vol. QE-13, no. 5, pp. 338-343, 1977.

[15] B. V. Rybakov, Yu. V. Demidenkov, S. G. Skrotskii, and A. M. Khromykh, "Amplitude and frequency characteristics of a ring laser," Soviet Physics JETP, vol. 30, no. 4, pp. 646-650, 1970.

[16] V. A. Markelov, "Frequency characteristic of a ring He-Ne laser," Izvestia Vuzov. Radiofizika, vol. 17, no. 11, pp. 1642-1648, 1974 (in Russian).

[17] A. M. Khromykh, "To the theory of a ring laser with nonstationary parameters," Elektron.Tekhn., Ser. Laser. Tekhn. Optoelektron., Issue 2(54), pp. 44-49, 1990 (in Russian).

[18] E. A. Bondarenko, "Expressions for counterpropagating waves beat frequency of uniformly rotating laser gyro," IEEE Central Ukraine Joint Chapter "Methods and Systems of Navigation and Motion Control" (MSNMC-2010). Kyiv, Ukraine. 13-16 October, 2010, pp. 142-145 (https://www.researchgate.net/publication/301748466).

[19] E. A. Bondarenko, "Frequency characteristic of a uniformly rotating laser gyroscope with differently amplified counterpropagating waves," Quantum Electron., vol. 32, no.2, pp. 160-164, 2002.

[20] E. A. Bondarenko, "Laser gyroscope dynamic equations. Formulae for calculation of active medium parameters and parameters of oppositely directed traveling waves linear coupling “, VII International Conference "Gyrotechnology, navigation, movement control and aerospace technic engineering". Kyiv, Ukraine. 23-24 April, 2009, pp. 115-124 (https://www.researchgate.net/publication/301748283).

[21] E. A. Bondarenko, "A laser gyro with a four-mirror square resonator: quantitative estimation of the dependence of the synchronisation zone parameters of the frequencies of counterpropagating waves on the activemedium gain," Quantum Electron., vol. 42, no. 5, pp. 465-470, 2012.

[22] E. A. Bondarenko, "A laser gyro with a four-mirror square resonator: formulas for simulating the dynamics of the synchronisation zone parameters of the frequencies of counterpropagating waves during the device operation in the self-heating regime," Quantum Electron., vol. 44, no. 4, pp. 364-370, 2014.

[23] Evgeny Bondarenko, "Polynomial model of the frequency characteristic of a quickly rotating laser gyro," ResearchGate, 2020 (https://www.researchgate.net/publication/343833982). 